\title{
Direct measurement of focusing fields in active plasma lenses
}

\author{
J.-H. Röckemann, ${ }^{1}$ L. Schaper, ${ }^{1}$ S. K. Barber ${ }^{2}$ N. A. Bobrova, ${ }^{3}$ G. Boyle, ${ }^{1}$ S. Bulanov, ${ }^{2}$ \\ N. Delbos, ${ }^{4}$ K. Floettmann, ${ }^{1}$ G. Kube, ${ }^{1}$ W. Lauth, ${ }^{5}$ W. P. Leemans, ${ }^{2}$ V. Libov, ${ }^{1,6}$ \\ A. R. Maier, ${ }^{4}$ M. Meisel, ${ }^{6}$ P. Messner, ${ }^{4}$ P. V. Sasorov, ${ }^{3}$ C. B. Schroeder, ${ }^{2}$ \\ J. van Tilborg, ${ }^{2}$ S. Wesch, ${ }^{1}$ and J. Osterhoff ${ }^{1, *}$ \\ ${ }^{1}$ Deutsches Elektronen-Synchrotron DESY, Notkestraße 85, 22607 Hamburg, Germany \\ ${ }^{2}$ Lawrence Berkeley National Laboratory, University of California, Berkeley, California 94720, USA \\ ${ }^{3}$ Keldysh Institute for Applied Mathematics, Moscow 125047, Russia \\ ${ }^{4}$ Center for Free-Electron Laser Science and Department of Physics, \\ Universität Hamburg, Luruper Chaussee 149, 22761 Hamburg, Germany \\ 5ohannes Gutenberg-Universität Mainz, Saarstraße 21, 55122 Mainz, Germany \\ ${ }^{6}$ Universität Hamburg, Mittelweg 177, 20148 Hamburg, Germany
}

(Received 24 June 2018; published 7 December 2018)

\begin{abstract}
Active plasma lenses have the potential to enable broad-ranging applications of plasma-based accelerators owing to their compact design and radially symmetric $\mathrm{kT} / \mathrm{m}$-level focusing fields, facilitating beam-quality preservation and compact beam transport. We report on the direct measurement of magnetic field gradients in active plasma lenses and demonstrate their impact on the emittance of a charged particle beam. This is made possible by the use of a well-characterized electron beam with $1.4 \mathrm{~mm}$ mrad normalized emittance from a conventional accelerator. Field gradients of up to $823 \mathrm{~T} / \mathrm{m}$ are investigated. The observed emittance evolution is supported by numerical simulations, which suggests the potential for conservation of the core beam emittance in such a plasma lens setup.
\end{abstract}

DOI: 10.1103/PhysRevAccelBeams.21.122801

\section{INTRODUCTION}

Laser wakefield accelerators (LWFAs) allow for the generation of extreme electric fields on the order of $100 \mathrm{GV} / \mathrm{m}$ for charged particle acceleration and can deliver beams of sub- $\mu \mathrm{m}$ normalized emittance $[1,2]$, multi-kA peak currents [3], and femtosecond pulse duration [4-6]. LWFAs have shown the capability to produce multi$\mathrm{GeV}$ electron beams in $\mathrm{cm}$-scale structures [7-9]. Their application to drive compact sources of coherent $\mathrm{x}$-ray beams $[10,11]$ and incoherent $\mathrm{MeV}$ photons [12], ultra-fast electron diffraction experiments $[13,14]$, and high-energy particle colliders [15] has been proposed and studied $[16,17]$. For all these applications small beam emittances are critical. Indeed, beams from plasma accelerators are susceptible to chromatic emittance growth in the drift following the acceleration section $[18,19]$. Thus, beam capturing within a few centimeters after the plasma exit is crucial for emittance preservation.

\footnotetext{
*Corresponding author. jens.osterhoff@desy.de

Published by the American Physical Society under the terms of the Creative Commons Attribution 4.0 International license. Further distribution of this work must maintain attribution to the author(s) and the published article's title, journal citation, and DOI.
}

In this context, conventional focusing optics face problems: Solenoids suffer from large chromaticity and weak focusing for relativistic beams owing to their $1 / \gamma^{2}$-scaling of the focusing strength, with the relativistic Lorentz factor $\gamma$. The more favorable $1 / \gamma$-scaling in combination with high field gradients $(\sim 500 \mathrm{~T} / \mathrm{m}$ for permanent magnets) of quadrupoles is put into perspective when considering that two quadrupoles need to be combined to achieve focusing in both transverse planes. Hence, quadrupoles, which are inherently defocusing in one plane, increase chromatic emittance growth in this plane dramatically [20].

Plasma-based beam focusing elements potentially offer an elegant solution to minimize chromatic emittance growth with their compact size, azimuthally symmetric focusing, and high magnetic field gradients. In passive plasma lenses electron beams are focused by means of a transverse plasma wakefield generated either by the beam itself, a second charged particle beam, or a high-intensity laser. Wake-based plasma lenses support linear focusing gradients for emittance preservation in the blow-out regime $[21,22]$ and have been proposed as final focusing optics to improve the luminosity of energy frontier linear colliders $[23,24]$. In contrast, the active plasma lens (APL) [25] realizes focusing fields employing a strong electric current co-linear with beam propagation with field gradients exceeding $3 \mathrm{kT} / \mathrm{m}$ [26]. Recent studies indicate that nonuniform current densities may form inside discharge 
capillary based APLs [27-31], leading to nonlinear magnetic field gradients and, subsequently, emittance deterioration $[32,33]$. In this work we report on a first direct measurement of the magnetic field distribution inside an APL and complement these results by experimentally detecting its influence on the emittance of a stable, wellcharacterized electron beam from a conventional accelerator. These studies are supported by simulations and show the potential for emittance preservation.

Active plasma lenses for electron beams typically consist of a gas-filled capillary with a circular cross-section of $\mathrm{mm}$-scale diameter and $\mathrm{cm}$-scale length machined into glass or sapphire. A multi-kV discharge voltage is applied to the capillary ends, leading to breakdown of the gas. Subsequently, a current is driven along the generated plasma column forming an azimuthal magnetic field. In the following, we assume an azimuthally symmetric current distribution $J(r)$, with $r$ denoting the radial position. Ampere's law provides the cylindrically symmetric magnetic field

$$
B_{\phi}(r) \cdot r=\mu_{0} \int_{0}^{r} J\left(r^{\prime}\right) r^{\prime} d r^{\prime}
$$

for $r<R$, with $R$ being the capillary radius and $\mu_{0}$ the vacuum permeability. The magnetic field distribution becomes $B_{\phi \text {,ideal }}(r)=\mu_{0} I_{0} r /\left(2 \pi R^{2}\right)$ in case of a uniform current distribution $J=I_{0} /\left(\pi R^{2}\right)$, with $I_{0}$ being the total current. Differentiating this expression yields the ideal magnetic field gradient

$$
g_{\text {ideal }}=\mu_{0} I_{0} /\left(2 \pi R^{2}\right) .
$$

\section{NONLINEAR MODEL OF ACTIVE PLASMA LENSES}

In general, Eq. (2) does not hold since the current distribution $J(r)$ is generally not uniform. A transverse temperature gradient forms due to cooling of the plasma at the capillary wall leading to a radially changing Ohmic resistance, a nonuniform current distribution, and a nonlinear magnetic field gradient. Figure 1 shows the result of a one-dimensional magnetohydrodynamic (MHD) simulation of a capillary of $R=0.5 \mathrm{~mm}$ radius filled with hydrogen of $n_{0}=10^{17} \mathrm{~cm}^{-3}$ molecular density traversed by a current of $I_{0}=364 \mathrm{~A}$ assuming a fixed electron temperature at the wall interface of $T^{*}=0.5 \mathrm{eV}$. The radial position is normalized to $R$, the magnetic field to $B_{\text {ideal }}$. Cases with $I_{0}=188 \mathrm{~A}$, and $740 \mathrm{~A}$ have also been simulated. The MHD modeling shows that for the currents used, the fraction of ionized hydrogen was well above $80 \%$.

An analytic model for the current distribution in a plasma lens was introduced in [32]. It is based on the Spitzer collisional model of plasma, in which the conductivity

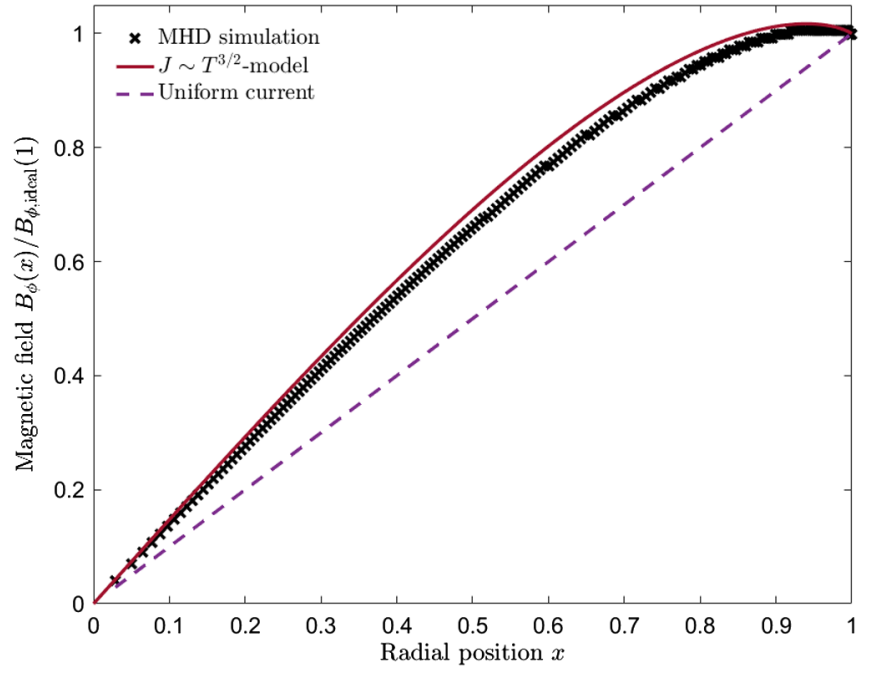

FIG. 1. MHD simulation results for a $R=0.5 \mathrm{~mm}$ gas column with $I_{0}=364$ A. The $J \sim T^{3 / 2}$-model is of the form of Eq. (7).

$\sigma$ depends on the plasma density $n_{e}$ and electron temperature $T_{e}$ via

$$
\sigma=\frac{32 \epsilon_{0}^{2}}{\ln \Lambda} \cdot \frac{\left(k_{B} T_{e}\right)^{3 / 2}}{e^{2} m_{e}^{1 / 2}},
$$

with $\lambda_{D}=\sqrt{\epsilon_{0} k_{B} T_{e} / n_{e} e^{2}}, \Lambda=n_{e} \lambda_{D}^{3}, k_{B}$ the Boltzmann constant, $\epsilon_{0}$ the vacuum permittivity, $e$ the electron charge, and $m_{e}$ the electron mass. The scaling of $\sigma$ is dominated by $T_{e}$ since $n_{e}$ appears only in the logarithm of $\Lambda$. Thus, the current density is dominated by the temperature $J(r)=$ $\sigma E \sim T_{e}^{3 / 2}$. Following the work of [27,32], the temperature distribution satisfies the heat flow equation

$$
\frac{1}{x} \frac{d}{d x}\left(x \frac{d u}{d x}\right)=-u^{3 / 7}
$$

in which $u^{2 / 7}=T_{e} / A$ with $A=\left(7 \sigma_{0} R^{2} E^{2} / 2 \kappa_{0}\right)^{1 / 2}$, $x=r / R$, and the thermal and electric conductivities were assumed to scale with $\kappa=\kappa_{0} T_{e}^{5 / 2}$ and $\sigma=\sigma_{0} T_{e}^{3 / 2}$, respectively. The boundary conditions satisfy $d T_{e}(x=0) /$ $d x=0$ and $T_{e}(x=1)=T^{*}$ with $T^{*}$ the temperature at the wall. The current distribution can be expressed as

$$
J(r)=\frac{I_{0} u(r)^{3 / 7}}{2 \pi R^{2} m_{I}},
$$

with $m_{I}=\int_{0}^{1} u^{3 / 7} x d x$. The central region $x<1$ can be written as

$$
\begin{aligned}
J(r)= & \frac{I_{0}}{\pi R^{2}}\left(\frac{u(0)^{3 / 7}}{2 m_{I}}\right)\left[1-\frac{3}{28} u(0)^{-4 / 7} x^{2}\right. \\
& \left.-\frac{15}{3136} u(0)^{-8 / 7} x^{4}\right],
\end{aligned}
$$


TABLE I. Comparison of measured and simulated gradients and emittances. The measured gradients are for an effective length of $L=7.5 \mathrm{~mm}$. Additionally, systematic uncertainties arising from the fringe fields are given. The emittance was simulated for $\sigma=154 \mu \mathrm{m}$ and a field in the form of the $J \sim T^{3 / 2}$ model (cf. Fig. 1).

\begin{tabular}{lcccccc}
\hline \hline$I_{0}$ & \multicolumn{3}{c}{$g_{\text {core }}(\mathrm{T} / \mathrm{m})$} & & \multicolumn{2}{c}{$\epsilon_{\mathrm{f}}(\mathrm{mm} \mathrm{mrad})$} \\
\cline { 2 - 4 } \cline { 6 - 7 }$(\mathrm{A})$ & Measurement & Fringe & $J \sim T^{3 / 2}$ & & Measurement & Simulation \\
\hline 188 & $238 \pm 9$ & \pm 17 & 223 & & $2.2 \pm 0.1$ & 2.5 \\
364 & $428 \pm 6$ & \pm 30 & 431 & & $3.7 \pm 0.1$ & 4.3 \\
740 & $823 \pm 8$ & \pm 59 & 876 & & $8.2 \pm 0.1$ & 8.4 \\
\hline \hline
\end{tabular}

and

$$
\begin{aligned}
B_{\phi}(x)= & \frac{\mu_{0} I_{0}}{2 \pi R} \cdot \frac{u(0)^{3 / 7}}{2 m_{I}} \cdot x \cdot\left[1-\frac{3}{56} u(0)^{-4 / 7} x^{2}\right. \\
& \left.-\frac{5}{3136} u(0)^{-8 / 7} x^{4}\right] .
\end{aligned}
$$

An important figure of merit for the linearity of an APL is its core linear magnetic field slope in comparison to the ideal magnetic field slope, defined as $\Delta g=g_{\text {core }} / g_{\text {ideal }}=\frac{u(0)^{3 / 7}}{2 m_{I}}$. The $\Delta g$-factor for the $J \sim T^{3 / 2}$-model in Fig. 1 is $\Delta g=1.48$. This corresponds to a cold wall boundary condition. The corresponding gradients are given in Table I.

\section{EXPERIMENTAL SETUP}

The APL in this experiment consisted of a $7 \mathrm{~mm}$ long capillary of $R=0.5 \mathrm{~mm}$ machined into a sapphire block. A continuous flow of hydrogen was supplied to the capillary at $4 \mathrm{mbar}$ backing pressure through two inlets of $R=$ $0.75 \mathrm{~mm}$ diameter situated $1.5 \mathrm{~mm}$ from the capillary ends leading to a molecular density of $n_{0}=10^{17} \mathrm{~cm}^{-3}$ inside the capillary. Copper electrodes on both sides connected a pulse-forming network [34] to the gas volume. A discharge voltage of 9-20 kV was applied which resulted in stable flat-top currents of 188-740 A arising $100 \mathrm{~ns}$ after the discharge trigger for a duration of $240 \mathrm{~ns}$. The electron beam traversed the APL 100 ns after the current plateaued. A schematic drawing of the APL inside the experimental setup is given in Fig. 2.

A race-track Microtron at the University of Mainz, the Mainz Microtron B (MaMi-B), was used for probing the magnetic field of the APL. MaMi-B was operated in a mode in which it delivered $10 \mathrm{~ns}$ long bunches with an average current of $100 \mu \mathrm{A}$, an energy of $855 \mathrm{MeV}$, and a normalized vertical emittance of $\epsilon_{\mathrm{i}}=1.37 \pm 0.01 \mathrm{~mm}$ mrad.

\section{EXPERIMENTAL RESULTS}

Direct measurements of the APL magnetic field gradients were performed by introducing a transverse offset of the APL with respect to the electron beam position, thus introducing a dipole kick to the beam. The centroid shifts and beam parameters of the MaMi-B beam were measured $d=25.3 \mathrm{~cm}$ downstream of the APL at screen S1 and averaged over 100 shots per offset position. The beam was focused into the APL in order to probe over the largest portion of the radius possible without beam clipping. Its dimensions at the capillary entrance were determined by backtracking the beam parameters based on the measurements at S1 without the plasma in its path. The beam size was calculated to be $80 \mu \mathrm{m} \mathrm{rms}$ in both planes. The offset was increased until clipping and charge loss of the beam became evident which resulted in a maximum offset of $350 \mu \mathrm{m}$. The resulting centroid shifts can be seen in Fig. 3 and are found to be linearly depending on the offset. The formation of fringe fields in APLs was discussed in [35]. Their influence on the emittance of a passing MaMi-type beam was simulated in ASTRA [36] and found to be negligible on the subpercent level. The longitudinal current ramp in the fringe fields was modeled after $I\left(z_{\text {edge }}\right)=$ $I_{0} /\left(1+\exp \left(4 z_{\text {edge }} / \sigma_{\text {ramp }}\right)\right)$, where $z_{\text {edge }}$ is the distance from the capillary end and $\sigma_{\text {ramp }}$ is the ramp taper parameter, as commonly used in conventional magnet optics. Owing to the fringe fields, the effective magnetic length $L=$ $L_{\text {capillary }}+2 \cdot L_{\text {fringe }}$ of the APL extends beyond the sapphire capillary itself. So the beam offset $\Delta\langle x\rangle$ is dependent on the

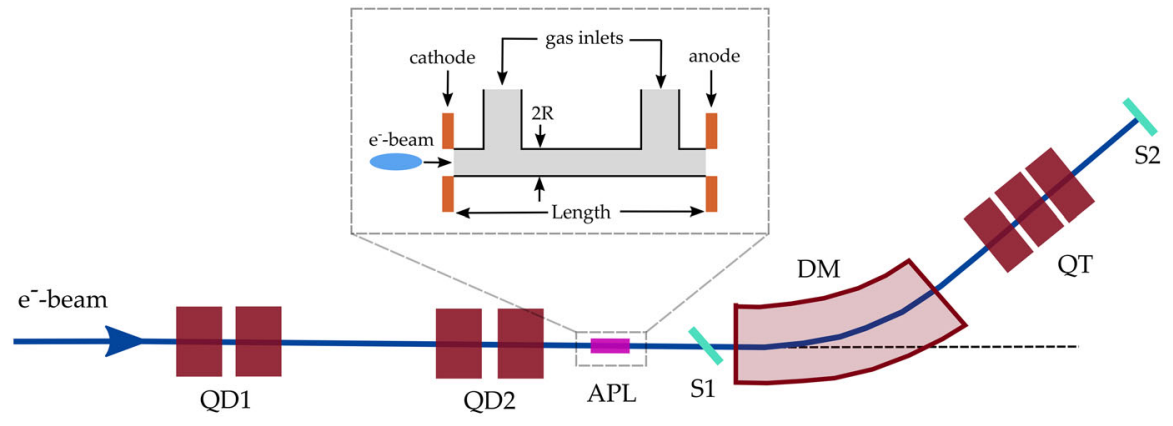

FIG. 2. Schematic of the accelerator beamline at MaMi-B. QD1: first quadrupole duplet; QD2: second quadrupole duplet; APL: active plasma lens; S1: screen used in the offset measurements; DM: dipole magnet; QT: quadrupole triplet used in the emittance measurements; S2: screen used in the emittance measurements. 


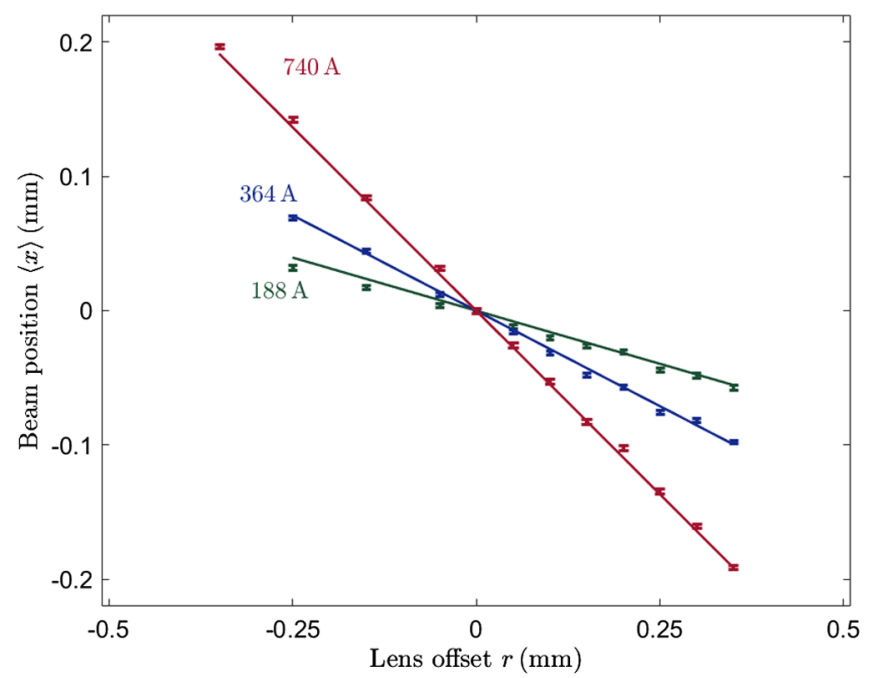

FIG. 3. Results from offset scan using $I_{0}=188$, 364, and 740 A of total current. The lines are linear fits to the data. The error bars include statistical fluctuations and underline the stability of the APL setup.

lens offset $r$ and effective length $L$ through the magnetic field

$$
\Delta\langle x\rangle=\frac{q \cdot d}{p} \int_{0}^{L} B_{\phi}(r) z d z
$$

in which $p$ is the particle momentum, $q$ its charge. To account for the additional uncertainty owing to the fringe field, the data in Fig. 3 was fitted with a linear model for the range of $L_{\text {fringe }} \leq 0.5 \mathrm{~mm}$ (which is well above the length found in [35]). The derived core gradients $g_{\text {core }}$ for $L_{\text {fringe }}=0.25 \mathrm{~mm}$ including the systematic uncertainty for $L_{\text {fringe }} \leq 0.5 \mathrm{~mm}$ can be found in Table I. The obtained magnetic field gradients are higher than Eq. (2) would predict from the measured discharge currents. They are, however, in good agreement with a $J \sim T^{3 / 2}$-model assuming a cold wall boundary condition with $\Delta g \sim 1.48$. It is noteworthy that the relative center-of-mass jitter of the MaMi-B beam was not affected by the APL even for the extreme case of $350 \mu \mathrm{m}$ offset (cf. Fig. 4). This means the magnetic field in the APL was highly reproducible, which may also be seen in the small error bars of the measured beam position in Fig. 3 .

A complementary way of probing the linearity of the magnetic field in the APL is measuring the emittance change of an electron beam after passage through the APL. Quadrupole scans were performed for different plasma lens settings in order to detect emittance change due to nonlinear field gradients. The currents used in the experiment were 188, 364, and $740 \mathrm{~A}$. The current amplitude had a jitter of $1.5 \mathrm{~A}$ rms in each case. This measurement technique requires the beamline upstream of the quadrupoles used for the scan to be stable. The here

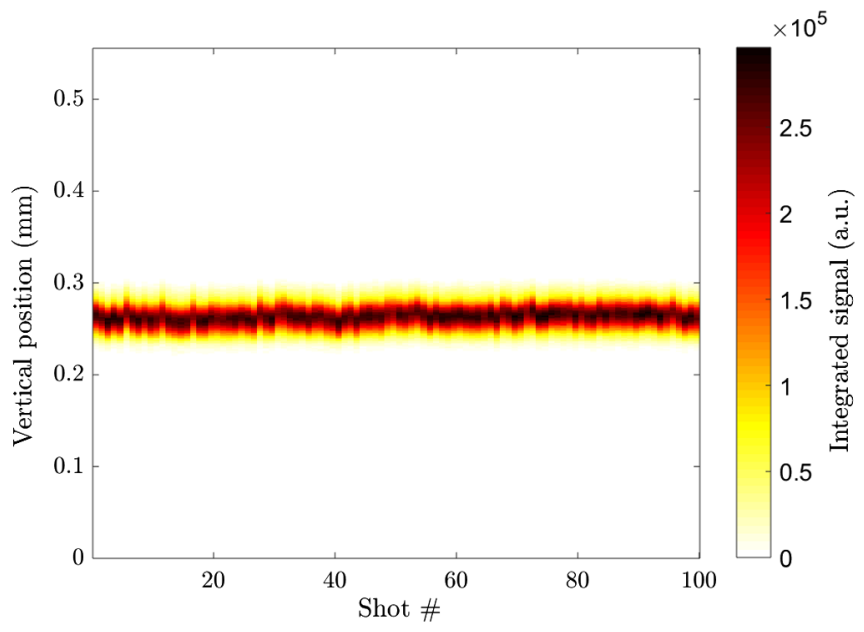

FIG. 4. Camera signal projected onto the vertical axis for 100 consecutive shots at $350 \mu \mathrm{m}$ APL offset. The excellent stability of MaMi was not measurably affected by the APL, which is indicated by the small shot-to-shot fluctuations of the signal.

reported APL stability greatly facilitated these emittance measurements and is reflected in the relatively low rms beam size variation during the scans of $<5 \%$ (100 shots were averaged per setting). In order to probe for nonlinearities over a large fraction of the capillary diameter an rms beam size of $\sigma_{y}=154_{-15}^{+5} \mu \mathrm{m}$ vertically and $\sigma_{x}=151_{-12}^{+2} \mu \mathrm{m}$ horizontally and a small divergence $\sigma_{x \prime, y \prime}<0.1 \mathrm{mrad}$ at the APL entrance were used for the emittance scans. The results of three quadrupole scans are shown in Table I. The measured beam sizes including fits can be found in Fig. 5. The optical system had a resolution of $20 \mu \mathrm{m}$ which was well suited for the $45 \mu \mathrm{m}$ of minimal beam size used in the scans.

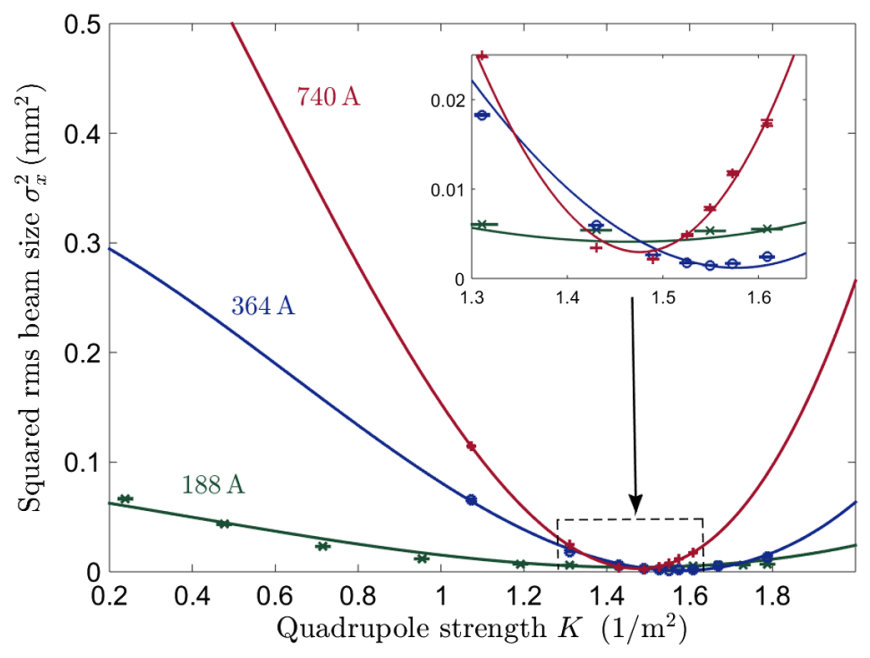

FIG. 5. Quadrupole scan results for the APL operated at 188, 364, and 740 A of total current. Error bars for beam size measurements are included. The fitted emittances can be found in Table I. 


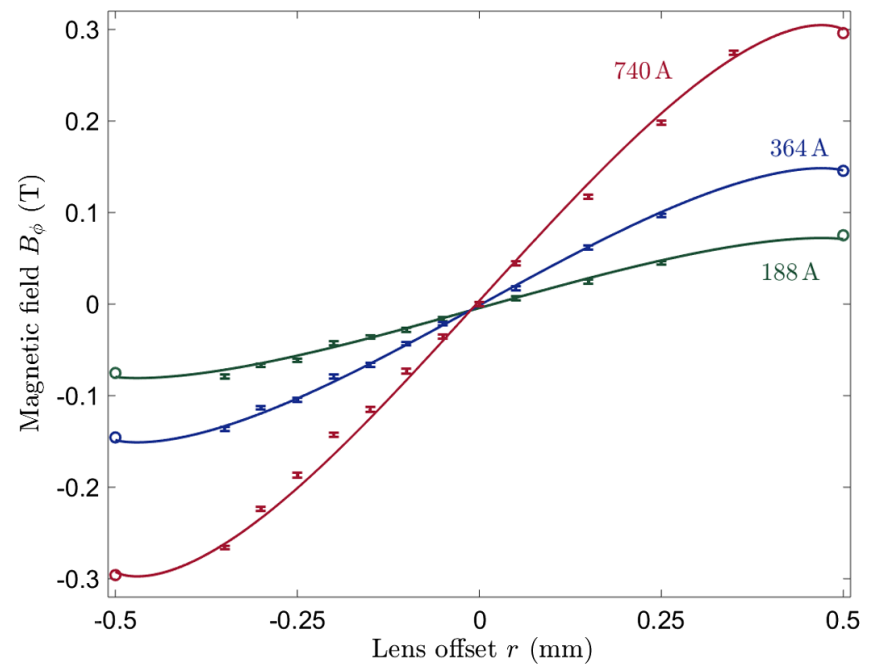

FIG. 6. Magnetic field strength in an $L=7.5 \mathrm{~mm}$ APL derived from the offset scan data in Fig. 3, for $r= \pm 0.5 \mathrm{~mm}$, obtained from measurements of $R$ and $I_{0}$ (circles). The sizes of the circular markers represent the rms error of the data points. The lines show the predicted behavior from the $J \sim T^{3 / 2}$-model.

At first glance, the offset measurements in Fig. 3 seem to imply, that field nonlinearity is not the root cause of the observed emittance degradation since the linear fits show excellent agreement with the data. Utilizing measurements of $R$ and $I_{0}$ allows us to derive the magnetic field strength at the wall through Eq. (1), showing that the linear behavior measured over the central $70 \%$ of the capillary diameter fails to describe the magnetic field for the entire capillary width. Since Eq. (1) is always fulfilled at the wall and $R$ and $I_{0}$ were measured with high precision, the derived magnetic field values have small errors of $< \pm 3 \%$. The $J \sim T^{3 / 2}$-model is in good agreement with all of the measurements including the detected emittance growth (see next section and Table I). Figure 6 shows the predicted behavior from the $J \sim T^{3 / 2}$-model assuming a cold wall boundary condition on top of the derived magnetic field values from the offset scan in Fig. 3 and as additional data points the magnetic field at the wall $(r= \pm R)$ from Eq. (1).

Other mechanisms for the emittance degradation such as self-wakefields and collisions fail to describe the observed dependence on total current which can readily be explained by a nonlinear field model. The driving of a self-wake can be neglected because of the low peak current used in the beam [37]. The emittance growth due to collisions can be estimated for: (a) multiple scattering in neutral background gas [38], and (b) transport in plasma $[39,40]$ including the stopping power effects of collisions with free, bound and screened electrons, and Bremsstrahlung $[40,41]$. For the parameters relevant to this work, the normalized emittance growth due to scattering is estimated to be $<0.05 \mathrm{~mm} \mathrm{mrad}$. Owing to the small energy spread of MaMi $\left(\sim 10^{-5}\right)$, chromatic effects were not relevant. The chromaticity introduced by the beam-plasma interaction was measured in the emittance measurements due to the dispersion introduced by the dipole in between the APL and the QMs used for the scans (cf. Fig. 2). No broadening of the energy spread was observed confirming the nonexistence of self-wakefields.

\section{SIMULATION RESULTS}

The emittance growth in an $L=7.5 \mathrm{~mm}$ long APL was simulated with the particle tracking code ASTRA. The field was modeled to be of the form given by the $J \sim T^{3 / 2}$ model. Transversally Gaussian shaped beams with rms beam size of $\sigma_{x, y}=154 \mu \mathrm{m}$ were assumed for the simulation. The measured emittance growth and the simulation results in Table I are in excellent agreement. This supports the conjecture that the $J \sim T^{3 / 2}$-model is a good approximation for the field behavior in the APL. Figure 7 shows particle tracking simulation results for different incoming rms beam sizes traversing the magnetic field distributions obtained from the $J \sim T^{3 / 2}$-model and $L=7.5 \mathrm{~mm}$ and $I_{0}=364 \mathrm{~A}$. They suggest a smaller beam size in the APL than used here is favorable to minimize emittance growth. The emittance growth is highly dependent on the incoming beam size and can be effectively eliminated for beams with $\sigma<75 \mu \mathrm{m}$ on the $1 \mathrm{~mm}$ mrad normalized emittance scale according to these numerical results. The data point shown in Fig. 7 corresponds to the measured emittance from Fig. 5 for 364 A.

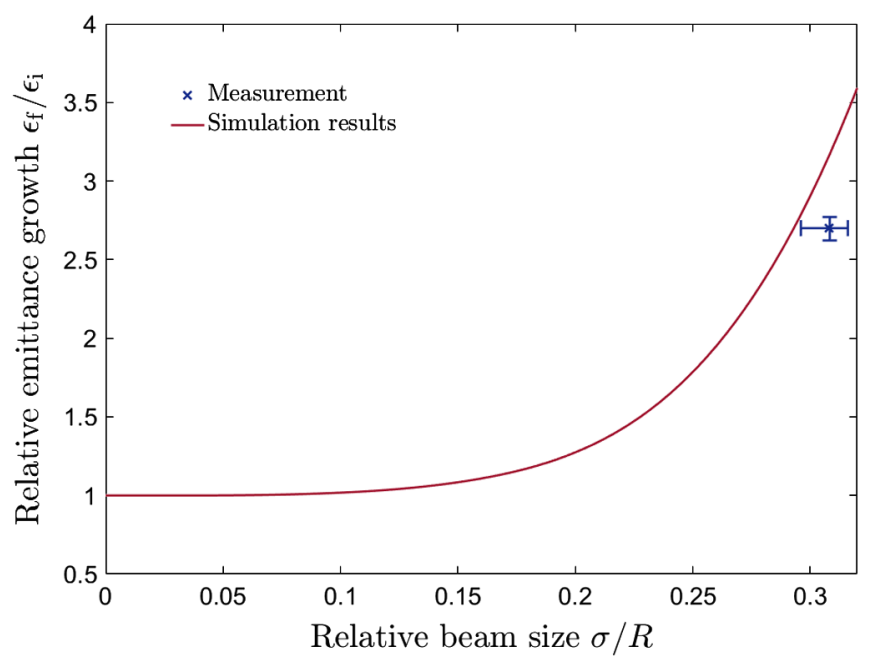

FIG. 7. Particle tracking simulation results for relative emittance degradation of a MaMi-like beam in dependence of incoming rms beam size for an APL with $R=0.5 \mathrm{~mm}$ and $I_{0}=364 \mathrm{~A}$. The measured emittance degradation for this setup is also shown. 


\section{CONCLUSION}

In summary, magnetic field gradients of a 1-mm diameter active plasma lens and the emittance change of a beam passing such a lens have been measured directly using the conventional accelerator Mainz Microtron. We observed excellent gradient stability. The measured gradient increase of $\Delta g \simeq 1.5$ showed a behavior predicted for a cold wall boundary condition $J \sim T^{3 / 2}$-model. The measured emittance change of a passing electron beam agrees with predictions made by magnetohydrodynamic simulations and particle tracking simulations using the measured gradient enhancement as input parameter. Furthermore, simulations suggest that using beams of an rms size smaller than $20 \%$ of the APL radius leads to emittance preservation on the mm mrad-level. Future studies will focus on mitigating emittance degradation further by manipulating the current density behavior in the APL by using different gas species and optimizing radii and current profiles.

\section{ACKNOWLEDGMENTS}

We acknowledge the support through the Helmholtz Virtual Institute VH-VI-503, the Helmholtz Matter and Technologies Accelerator Research and Development program, the Helmholtz IuVF ZT-0009 program, and the U.S. Department of Energy under Contract No. DEAC02-05CH11231.

[1] S. Barber et al., Measured Emittance Dependence on the Injection Method in Laser Plasma Accelerators, Phys. Rev. Lett. 119, 104801 (2017).

[2] R. Weingartner et al., Ultralow emittance electron beams from a laser-wakefield accelerator, Phys. Rev. ST Accel. Beams 15, 111302 (2012).

[3] C. G. R. Geddes, Cs. Toth, J. van Tilborg, E. Esarey, C. B. Schroeder, D. Bruhwiler, C. Nieter, J. Cary, and W. P. Leemans, High-quality electron beams from a laser wakefield accelerator using plasma-channel guiding, Nature (London) 431, 538 (2004).

[4] O. Lundh et al., Few femtosecond, few kiloampere electron bunch produced by a laser-plasma accelerator, Nat. Phys. 7, 219 (2011).

[5] K. Zeil, J. Metzkes, T. Kluge, M. Bussmann, T. E. Cowan, S. D. Kraft, R. Sauerbrey, and U. Schramm, Direct observation of prompt pre-thermal laser ion sheath acceleration, Nat. Commun. 3, 874 (2012).

[6] A. Buck, M. Nicolai, K. Schmid, C. M. S. Sears, A. Sävert, J. M. Mikhailova, F. Krausz, M. C. Kaluza, and L. Veisz, Real-time observation of laser-driven electron acceleration, Nat. Phys. 7, 543 (2011).

[7] H. T. Kim, K. H. Pae, H. J. Cha, I. J. Kim, T. J. Yu, J. H. Sung, S. K. Lee, T. M. Jeong, and J. Lee, Enhancement of Electron Energy to the Multi-GeV Regime by a Dual-Stage Laser-Wakefield Accelerator Pumped by Petawatt Laser Pulses, Phys. Rev. Lett. 111, 165002 (2013).
[8] X. Wang et al., Quasi-monoenergetic laser-plasma acceleration of electrons to $2 \mathrm{GeV}$, Nat. Commun. 4, 1988 (2013).

[9] W. Leemans et al., Multi-GeV Electron Beams from Capillary-Discharge-Guided Subpetawatt Laser Pulses in the Self-Trapping Regime, Phys. Rev. Lett. 113, 245002 (2014).

[10] A. R. Maier, A. Meseck, S. Reiche, C. B. Schroeder, T. Seggebrock, and F. Grüner, Demonstration Scheme for a Laser-Plasma-Driven Free-Electron Laser, Phys. Rev. X 2, 031019 (2012).

[11] Z. Huang, Y. Ding, and C. B. Schroeder, Compact X-ray Free-Electron Laser from a Laser-Plasma Accelerator Using a Transverse-Gradient Undulator, Phys. Rev. Lett. 109, 204801 (2012).

[12] S. Chen et al., MeV-Energy X Rays from Inverse Compton Scattering with Laser-Wakefield Accelerated Electrons, Phys. Rev. Lett. 110, 155003 (2013).

[13] P. Catravas, E. Esarey, and W. P. Leemans, Femtosecond X-rays from Thomson scattering using laser wakefield accelerators, Meas. Sci. Technol. 12, 1828 (2001).

[14] F. V. Hartemann, D. J. Gibson, W. J. Brown, A. Rousse, K. Ta Phuoc, V. Mallka, J. Faure, and A. Pukhov, Compton scattering $\mathrm{X}$-ray sources driven by laser wakefield acceleration, Phys. Rev. ST Accel. Beams 10, 011301 (2007).

[15] C. Schroeder, E. Esarey, C. Geddes, C. Benedetti, and W. Leemans, Physics considerations for laser-plasma linear colliders, Phys. Rev. ST Accel. Beams 13, 101301 (2010).

[16] Y. Glinec et al., High-Resolution $\gamma$-Ray Radiography Produced by a Laser-Plasma Driven Electron Source, Phys. Rev. Lett. 94, 025003 (2005).

[17] N. D. Powers, I. Ghebregziabher, G. Golovin, C. Liu, S. Chen, S. Banerjee, J. Zhang, and D. P. Umstadter, Quasimonoenergetic and tunable X-rays from a laser-driven Compton light source, Nat. Photonics 8, 28 (2014).

[18] K. Floettmann, Some basic features of the beam emittance, Phys. Rev. ST Accel. Beams 6, 034202 (2003).

[19] T. Mehrling, J. Grebenyuk, F. Tsung, K. Floettmann, and J. Osterhoff, Transverse emittance growth in staged laserwakefield acceleration, Phys. Rev. ST Accel. Beams 15, 111303 (2012).

[20] C. Lindstrøm and E. Adli, Design of general apochromatic drift-quadrupole beam lines, Phys. Rev. Accel. Beams 19, 071002 (2016).

[21] N. Barov and J. Rosenzweig, in Proceedings of the 15th Particle Accelerator Conference, PAC-1993, Washington, DC, 1993 (IEEE, New York, 1993), Vol. 4, pp. 2623-2625.

[22] J. B. Rosenzweig, B. Breizman, T. Katsouleas, and J. J. Su, Acceleration and focusing of electrons in two-dimensional nonlinear plasma wake fields, Phys. Rev. A 44, R6189 (1991).

[23] P. Chen, A possible final focussing mechanism for linear colliders, Part. Accel. 20, 171 (1987).

[24] J.S. Ng et al., Observation of plasma focusing of a 28.5 GeV positron beam, Phys. Rev. Lett. 87, 24 (2001).

[25] E. Forsyth et al., The brookhaven-columbia plasma lens, Meas. Sci. Technol. 12, 872 (1965). 
[26] J. van Tilborg et al., Active Plasma Lensing for Relativistic Laser-Plasma-Accelerated Electron Beams, Phys. Rev. Lett. 115, 184802 (2015).

[27] N. A. Bobrova, A. A. Esaulov, J.-I. Sakai, P. V. Sasorov, D. J. Spence, A. Butler, S. M. Hooker, and S. V. Bulanov, Simulations of a hydrogen-filled capillary discharge waveguide, Phys. Rev. E 65, 016407 (2001).

[28] D. Spence and S. M. Hooker, Investigation of a hydrogen plasma waveguide, Phys. Rev. E 63, 015401 (2000).

[29] A. Butler, D. Spence, and S. M. Hooker, Guiding of 35 TW laser pulses in ablative capillary discharge waveguides, Phys. Rev. Lett. 89, 185003 (2002).

[30] C. McGuffey, M. Levin, T. Matsuoka, V. Chvykov, G. Kalintchenko, P. Rousseau, V. Yanovsky, A. Zigler, A. Maksimchuk, and K. Krushelnick, Guiding of 35 TW laser pulses in ablative capillary discharge waveguides, Phys. Plasmas 16, 113105 (2009).

[31] A. Gonsalves et al., Demonstration of a high repetition rate capillary discharge waveguide, J. Appl. Phys. 119, 033302 (2016).

[32] J. van Tilborg et al., Nonuniform discharge currents in active plasma lenses, Phys. Rev. Accel. Beams 20, 032803 (2017).
[33] R. Pompili et al., Experimental characterization of active plasma lensing for electron beams, Appl. Phys. Lett. 110, 104101 (2017).

[34] P. G. Donald, Pulse forming network, US Patent 2,769,903 (1956).

[35] G. Bagdasorov et al., Laser beam coupling with capillary discharge plasma for laser wakefield acceleration applications, Phys. Plasmas 24, 083109 (2017).

[36] K. Floettmann, Astra particle tracking code. www.desy.de/ $\sim$ mpyflo.

[37] C. A. Lindstrøm and E. Adli, Analytic plasma wakefield limits for active plasma lenses, arXiv:1802.02750.

[38] M. Reiser, Theory and Design of Charged Particle Beams (John Wiley \& Sons, New York, 2008).

[39] B. Montague and W. Schnell, Multiple scattering and synchrotron radiation in the plasma beat-wave accelerator, AIP Conf. Proc. 130, 146 (1985).

[40] S. Humphries, Charged Particle Beams (Courier Corporation, New York, 2013).

[41] M. Touati, J.-L. Feugeas, Ph. Nicolaï, J. J. Santos, L. Gremillet, and V.T. Tikhonchuk, A reduced model for relativistic electron beam transport in solids and dense plasmas, New J. Phys. 16, 073014 (2014). 